\title{
Identification of strain-rate sensitivity parameters of steel sheet by genetic algorithm optimisation
}

\author{
G. Belingardi, G. Chiandussi \& A. Ibba \\ Dipartimento di Meccanica, Politecnico di Torino, Italy
}

\begin{abstract}
The influence of the loading speed on mechanical response of structural materials can be accounted by means of strain-rate sensitivity parameters. The aim of the present work is to show a numerical technique based on an inverse approach to determine strain-rate sensitivity parameters of steels for car body constructions. This technique is based on the numerical simulation of a simple test according to the ASTM D5420/96 standard by means of a finite element explicit code. The test consists of a falling tup with a spherical head impacting on a thin sheet. Some experimental tests are conducted at different speeds, from quasi-static to impact loading conditions, on a specimen made of XE280P steel. A series of simulations are performed, changing the strain-rate sensitivity parameters in each run according to a genetic algorithm strategy. The strain-rate parameters that lead to the best fit of the experimental load-displacement curve with the numerical result are the assumed material characteristic parameters. The Cowper-Symonds and Johnson-Cook strain rate models have been taken into consideration.
\end{abstract}

Keywords: strain-rate sensitivity, optimisation, genetic algorithm, finite element analysis.

\section{Introduction}

Since the second half of the past century, research in the automotive industry focused on safety improvement. Increasing customer interest about passive safety and the ever stricter regulations, both in the US and EU, pushed towards more reliable vehicle structure design and a deeper insight into material behaviour. The finite element method applied to crash simulations with explicit 
codes and the increasing computing capability showed the way to new possibilities for prediction of passive safety performance of vehicles. But these opportunities have to be accompanied by adequate information on material mechanical behaviour and by proper material modelling: both are fundamental for correct numerical simulation. As a consequence material characterisation and model identification through experimental tests have gained a primary role.

One of the most critical uncertainties in crash simulations relates to the influence of the loading speed on the material mechanical characteristics. This influence can be accounted by means of the strain-rate sensitivity parameters that are substantial for most materials. Large strain-rate sensitivity of low-carbon steels, such as the deep-drawing steels of most automotive body, is very well known.

Strain rate sensitivity characterisation is quite complex because of the variety of tests that could be performed and the difficulty in data acquisition. Uniaxial tensile tests can be performed only at low strain rate values with traditional hydraulic testing machines, moreover it is not possible to perform tests at strictly constant strain rates and often the strain rate is not uniform on the specimen. Other type of tests and testing machines, as for example the split Hopkinson pressure bar, have been developed to characterise strain rate sensitivity of materials. The instrumented drop dart test has proven to give interesting opportunities in pointing out the material sensitivity to the strain rate and has been successfully used for this type of characterisation.

In the present work an optimisation technique, based on a genetic algorithm, is applied in order to determine parameters for strain-rate sensitivity models for a high strength steel to be adopted in car body constructions. It is based on the numerical simulation, by means of a finite element explicit code model, of the simple drop dart test performed at different strain rates and the interactive changing of material parameters until an optimum fit of experimental data is reached. The Johnson-Cook and the Cowper-Symonds strain rate sensitivity models are analysed in the present work. The considered material is the steel XE280P laminated in sheets of $1.5 \mathrm{~mm}$ thickness.

\section{Experimental tests and numerical models}

Quasi-static uniaxial tensile tests and a series of simple bending tests at different strain rates were performed to characterise the XE280P steel sheets and to identify parameters of models. Both types of test are necessary because they involve different mechanisms of deformation and help the stability and completeness of the identification.

The uniaxial tensile tests were performed by means of a general purpose hydraulic testing machine (DARTEC HA100). The usual dog bone shaped specimens were manufactured from a laminated sheet of $1.5 \mathrm{~mm}$ thickness. The obtained true stress vs. true strain curve is fundamental for the implementation of the material behaviour in FE codes and was used for a first direct characterisation (fig. 1). 


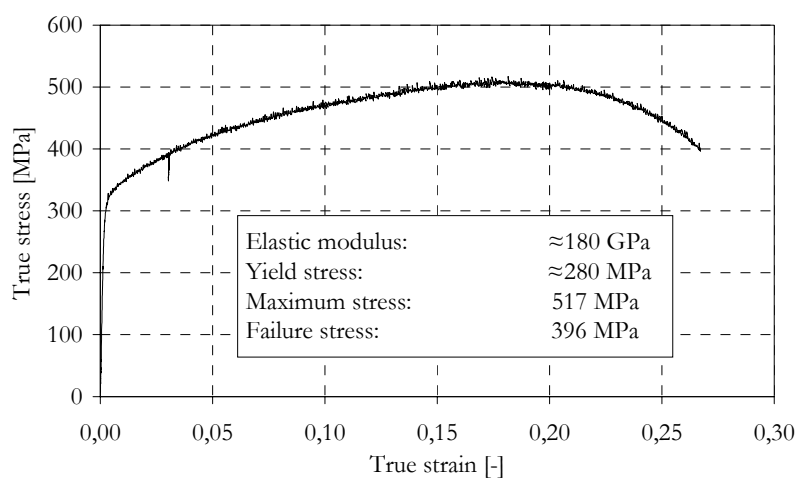

Figure 1: Experimental stress-strain curve from quasi-static uniaxial tensile test.

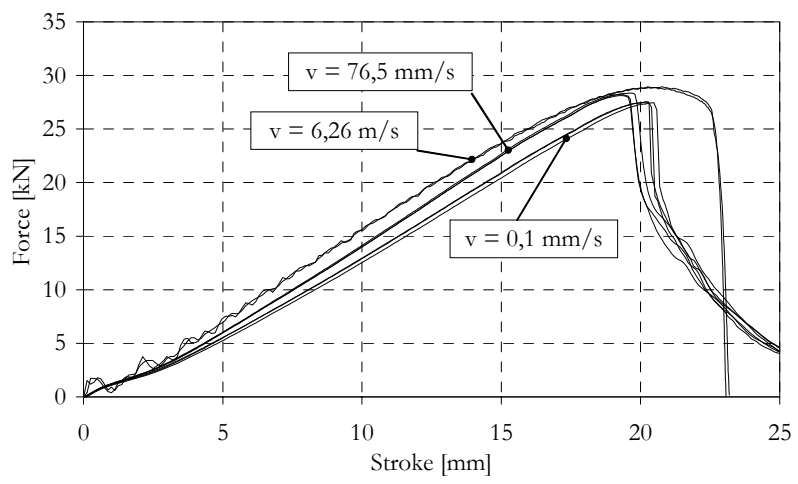

Figure 2: Experimental force-displacement curves of bending tests performed at different loading speeds.

Bending tests based on the ASTM D5420/96 standard were performed in order to characterise the strain rate sensitivity parameters of the steel. The test consists on a falling tup with a spherical head impacting a steel sheet. The specimens are square shaped but are completely constrained on a circumference of diameter $76 \mathrm{~mm}$ by means of a blank-holder plate. The tests are conducted at different speeds, from quasi-static loading conditions by means of the hydraulic testing machine, to impact loading conditions, by means of a drop-dart testing machine characterised by settable impact speed up to $6.26 \mathrm{~m} / \mathrm{s}$. The falling mass (including the dart) is $20 \mathrm{~kg}$. Each test gives a load-stroke curve. Force values are measured with a load cell placed at the dart head while displacement is obtained by LVDT direct measurement for the quasi-static testing conditions and by double integration of the acceleration in time for the impact testing conditions. Direct speed measurements are used in impact test for confirmation. Figure 2 shows some experimental curves. Modifications induced by the load application speed are evident. Numerical simulations of the bending tests were 
carried out by means of the code RADIOSS. Different meshes and element formulations were tested, and both demonstrated to have little influence on the final results. The default Belytschko formulation with global plasticity algorithm of Radioss was chosen for shell elements. Two models were used: a simplified model with a complete constrain on the clamping circumference and a more complete model with rigid plates holding the square specimen (fig. 3). Both models take advantage of the symmetry of the system.
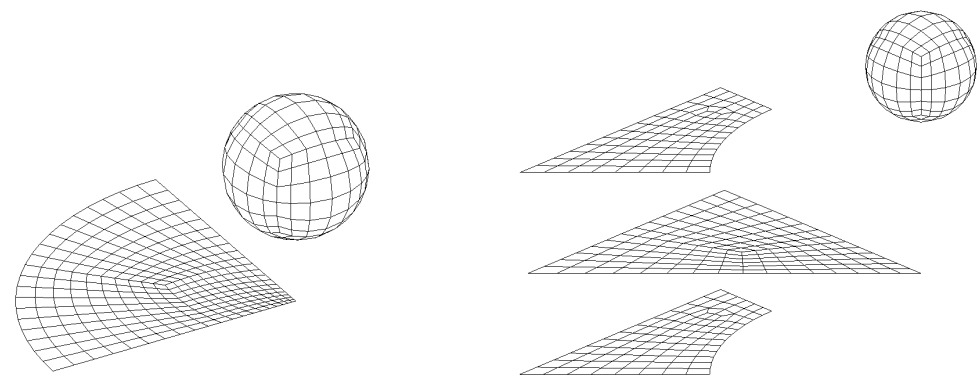

Figure 3: On the left: simplified model of the bending test; the external circumference is completely constrained. On the right: exploded view of the complete model with the specimen holding plates.

Beyond the yield stress, the static plastic behaviour of the material is modelled, as usual, by the following Hollomon formulation:

$$
\sigma_{\mathrm{s}}=\mathrm{A}+\mathrm{B} \varepsilon_{\mathrm{pl}}{ }^{\mathrm{n}}
$$

where $\varepsilon_{p l}$ is the plastic component of the total strain, while $A, B$ and $n$ are material constants. For the dynamic behaviour two strain rate sensitivity models have been considered. Both models modify the static stress-strain curve by adding a multiplying factor depending on the strain rate and both models are characterised by the presence of two parameters that have to be identified. The Cowper-Symonds model has the following formulation:

$$
\sigma=\sigma_{\mathrm{s}}(\varepsilon) \cdot\left(1+\left(\frac{\dot{\varepsilon}}{\mathrm{D}}\right)^{1 / \mathrm{p}}\right)
$$

where $\sigma_{s}(\varepsilon)$ is the static stress and $D$ and $p$ are material constants. The JohnsonCook model includes a further factor to account for the temperature dependence that is not considered in this work:

$$
\sigma=\sigma_{\mathrm{s}}(\varepsilon) \cdot\left(1+\mathrm{C} \ln \left(\frac{\dot{\varepsilon}}{\dot{\varepsilon}_{0}}\right)\right) \cdot\left(1-\frac{\mathrm{T}}{\mathrm{T}_{\mathrm{m}}}\right)
$$

where $\mathrm{C}$ and $\dot{\varepsilon}_{0}$ are the material constants. 


\section{Identification by genetic algorithm optimisation}

The identification of the static and strain rate sensitivity parameters was performed by means of an optimisation technique. A set of simulations were executed, changing the values assigned to the material characterising parameters in each run according to a genetic algorithm strategy. For each run a complete generation of a defined number of models was analysed. The parameters that lead to the best fit of the numerical result with respect to the experimental result have been selected as the optimal material model parameters. In particular the following objective function to be minimised was used:

$$
F\left(\operatorname{par}_{1}, \ldots, \operatorname{par}_{\mathrm{q}}\right)=\sqrt{\frac{\sum_{\mathrm{i}=1}^{\mathrm{N}}\left(\mathrm{y}_{\text {exp }, \mathrm{i}}-\mathrm{y}_{\text {mod,i }}\right)^{2}}{\mathrm{~N}}}
$$

where $\operatorname{par}_{q}$ are the parameter values to be identified, $y_{\text {exp }, i}$ and $y_{\text {mod, } i}$ are the experimental and numerical responses respectively, and $\mathrm{N}$ is the number of curve points. The objective function was evaluated on the stress-strain curve for the uniaxial tensile test and on the force-displacement curve for the bending tests. In both cases curve data up to the maximum of, respectively, the stress or the force were considered.

The genetic algorithm is defined by the mechanisms of selection, recombination and mutation. The selection is used to choose the parent models and is performed randomly by means of probability functions derived from the model fitness: the higher is the model fitness the higher is the probability to be chosen as parent of the new generation. The model fitness is defined as the difference between the value of the objective function for the worst model of the generation and the value of the objective function for the model itself. The selection is limited to the models that constitute the $50 \%$ of the cumulated fitness, decreasingly ordered. The recombination is the main feature of the genetic algorithm. It defines the son models by combining different parent models:

$$
\mathrm{x}_{\mathrm{a}}=\left(1-\mu_{\alpha}\right) \cdot \mathrm{x}_{\alpha}+\mu_{\beta} \cdot \mathrm{x}_{\beta} ; \mathrm{x}_{\mathrm{b}}=\mu_{\alpha} \cdot \mathrm{x}_{\alpha}+\left(1-\mu_{\beta}\right) \cdot \mathrm{x}_{\beta}
$$

Two son models $\left(\mathrm{x}_{\mathrm{a}}, \mathrm{x}_{\mathrm{b}}\right)$ are generated from two parent models $\left(\mathrm{x}_{\alpha}, \mathrm{x}_{\beta}\right) . \mu_{\alpha}$ and $\mu_{\beta}$ are random numbers generated by a normal probability function with null mean value. Finally a mutation, limited to $20 \%$ of the admissibility domain, is imposed and the generated models are computed by FE simulations. The best fitness model selected among the previous generation models and the new generated model constitute the present generation.

Parameters identification was also performed by a response surface and a gradient method in order to evaluate, by comparison, the performance of the genetic algorithm. 


\subsection{Static model parameters identification}

Parameter identification of the static model of eqn. (1) by means of uniaxial tensile test does not need FE simulations. The good fitting of the model obtained by genetic algorithm is shown in fig. 4, together with the objective function level curves on the $A-n$ parameter plane. These curves highlight a large region of $A$ and $n$ values with a nearly constant value of the objective function that means possible instability in parameter identification. Multiple optimum couples of values of these parameters are possible and for this reason a fixed $A$ parameter defined by a direct identification have been used.
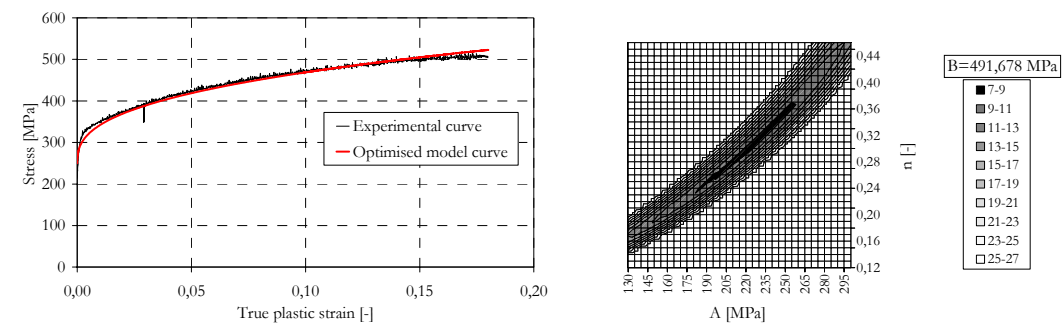

Figure 4: Identified model by uniaxial tensile test and level curves of the objective function on the $A-n$ parameters plane.

The bending test performed at low speed can also be used for the static parameters identification. In this case different and not uniform mechanisms of deformation are involved and FE simulations are needed to have the numerical force-displacement curve to be compared with the experimental one.
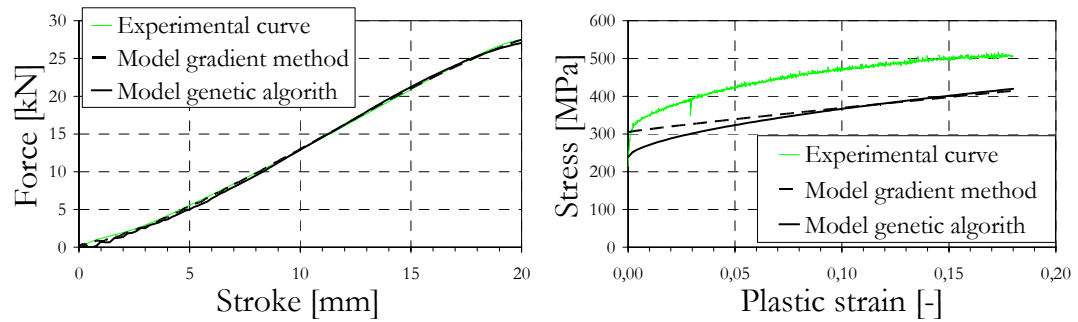

Figure 5: Simulated static bending test and stress-strain models obtained by optimisation with bending test and simplified FE model.

The parameters identified by means of the genetic algorithm and of the gradient method based on the simplified structural model of the bending test (fig. 3(a)) bring to the force-displacement and stress-strain curves shown in fig. 5. Both optimisation method work well to fit the experimental curve of the bending test, but the identified parameters are rather different from each other and the corresponding stress-strain characteristics are rather far from the experimental one. This confirms the possible criticality of the identification 
method that could lead to the same well simulated curves with different parameter values. Moreover it shows that the FE model of the bending test could be not sufficiently accurate, both for what concern the mesh refinement and for what concern the through thickness integration.
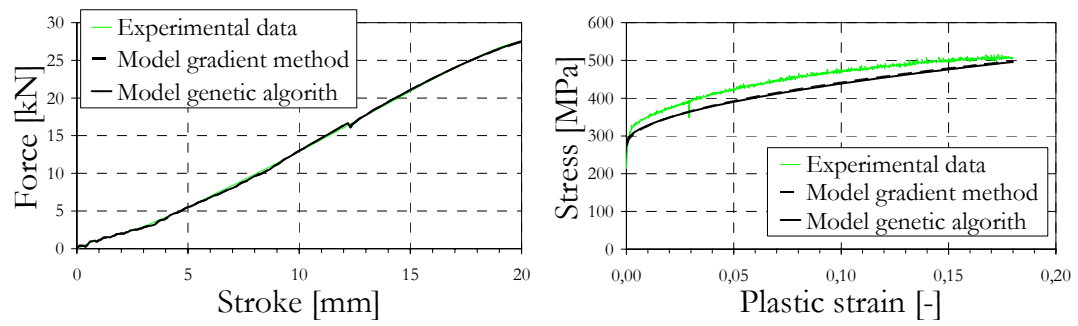

Figure 6: $\quad$ Simulated static bending test and stress-strain models obtained by optimisation with bending test and complete FE model.

The use of the complete structural model (fig. 3(b)) for the identification leads to the results shown in fig. 6. A more accurate solution is obtained: the material model fits better the experimental stress-strain curve of the tensile test, while keeping the same quality of fitness for the force-displacement curve of bending test. All the identified parameters are summarised in table 1 .

Table 1: Optimal values for the static model.

\begin{tabular}{|c|c|c|c|c|c|c|}
\cline { 2 - 7 } \multicolumn{1}{c|}{} & \multicolumn{2}{c|}{$\begin{array}{c}\text { Uniaxial tensile } \\
\text { test }\end{array}$} & \multicolumn{2}{c|}{$\begin{array}{c}\text { Bending test } \\
\text { Simplified model }\end{array}$} & \multicolumn{2}{c|}{$\begin{array}{c}\text { Bending test } \\
\text { Complete model }\end{array}$} \\
\cline { 2 - 7 } \multicolumn{1}{c|}{} & Genetic & Gradient & Genetic & Gradient & Genetic & Gradient \\
\hline $\mathrm{A}[\mathrm{MPa}]$ & 241.7 & 213.5 & 238.7 & 305.2 & 282.4 & 273.1 \\
\hline $\mathrm{B}[\mathrm{MPa}]$ & 482.5 & 491.7 & 499.0 & 521.1 & 532.1 & 528.4 \\
\hline $\mathrm{N}[-]$ & 0.3298 & 0.2857 & 0.5913 & 0.9116 & 0.5323 & 0.4967 \\
\hline $\mathrm{F}[\mathrm{MPa}, \mathrm{kN}]$ & 8.328 & 8.186 & 0.361 & 0.179 & 0.173 & 0.185 \\
\hline
\end{tabular}

\subsection{Strain rate sensitivity model identification}

For the identification of the strain rate sensitivity parameters drop tower tests were performed with two different falling heights of the dart: $1 \mathrm{~m}$ and $2 \mathrm{~m}$. In order to achieve a more suitable solution the static parameters identified by means of the uniaxial tensile test were used and only the strain rate parameters were searched with the described optimisation procedure. The objective function to be minimised take into account the force-displacement curves of both test conditions:

$$
\mathrm{F}\left(\mathrm{p}_{1}, \mathrm{p}_{2}\right)=\frac{1}{2} \sqrt{\frac{\sum_{\mathrm{i}=1}^{\mathrm{N}_{1}}\left(\mathrm{~F}_{\exp 1, \mathrm{i}}-\mathrm{F}_{\bmod 1, \mathrm{i}}\right)^{2}}{\mathrm{~N}_{1}}}+\frac{1}{2} \sqrt{\frac{\sum_{\mathrm{i}=1}^{\mathrm{N}_{2}}\left(\mathrm{~F}_{\exp 2, \mathrm{i}}-\mathrm{F}_{\bmod 2, \mathrm{i}}\right)^{2}}{\mathrm{~N}_{2}}}
$$




\subsubsection{Cowper-Symonds model}

The identification of Cowper-Symonds parameters by genetic algorithm was performed by means of 16 models for each generation. For the complete model, the evolution of objective function values for each generation in the best and worst cases are shown in fig. 7(a). In figure 7(b) the values obtained by the gradient method for each iteration are shown for effectiveness comparison. The genetic algorithm optimisation shows a more rapid convergence, even if a greater amount of simulations were used. The identified parameters and the final objective function values are summarised in table 2 . The force-displacement curves derived from the simulations with the optimised complete model are shown and compared to the experimental ones in fig. 8 .
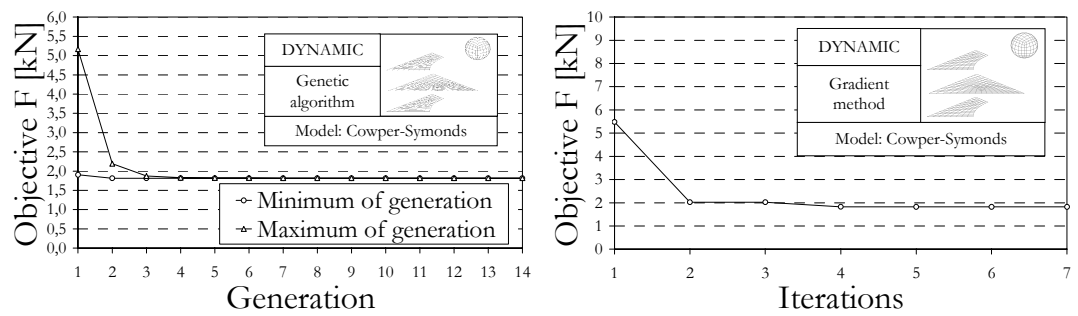

Figure 7: The objective function behaviour with the genetic algorithm and the gradient method.

Table 2: Optimal values of the Cowper-Symonds strain rate sensitivity model.

\begin{tabular}{|c|c|c|c|c|}
\cline { 2 - 5 } \multicolumn{1}{c|}{} & \multicolumn{2}{c|}{ Complete model } & \multicolumn{2}{c|}{ Simplified model } \\
\cline { 2 - 5 } \multicolumn{1}{c|}{} & Genetic & Gradient & Genetic & Gradient \\
\hline $\mathbf{D ~}\left[\mathbf{s}^{-1}\right]$ & 4987 & 3013 & 2399 & 3006 \\
\hline $\mathbf{p ~ [ - ]}$ & 1.619 & 1.503 & 1.329 & 1.416 \\
\hline $\mathbf{F}[\mathbf{k N}]$ & 1.817 & 1.822 & 1.187 & 1.209 \\
\hline
\end{tabular}

\subsubsection{Johnson-Cook model}

The identified parameters of the Johnson-Cook model by means of the genetic algorithm and the gradient method, with both the simplified and complete models are summarised in table 3. Five models were computed for each generation of the genetic algorithm optimisation. Force-displacement curves obtained by simulations with the complete model optimised by the genetic algorithm are shown and compared to the experimental ones in fig. 9.

\section{Conclusions}

A genetic algorithm optimisation procedure was applied to the identification of strain rate sensitivity models and proved to be effective. Compared to an optimisation procedure based on the gradient method it reached a better quality result in less iterations, even if the number of simulations to be run could be high. 


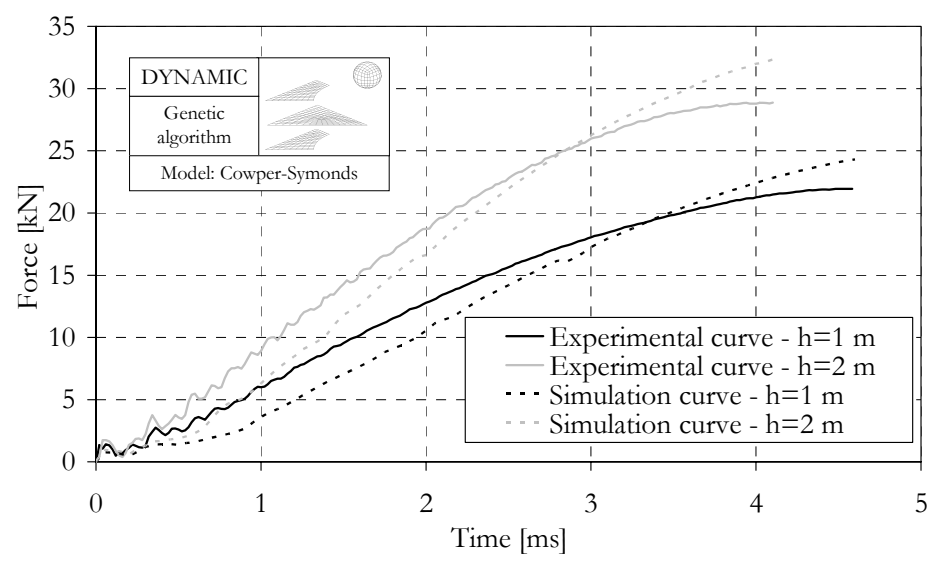

Figure 8: Comparison between experimental force-time curves of dynamic bending tests and simulations with Cowper-Symonds model.

Table 3: Optimal values for the Johnson-Cook strain rate sensitivity model.

\begin{tabular}{|c|c|c|c|c|}
\cline { 2 - 5 } \multicolumn{1}{c|}{} & \multicolumn{2}{c|}{ Complete model } & \multicolumn{2}{c|}{ Simplified model } \\
\cline { 2 - 5 } \multicolumn{1}{c|}{} & Genetic & Gradient & Genetic & Gradient \\
\hline$\dot{\varepsilon}_{0}\left[\mathbf{s}^{-1}\right]$ & \multicolumn{2}{c|}{1.00} & \multicolumn{2}{c|}{1.00} \\
\hline $\mathbf{C}[-]$ & 0,01149 & 0,01223 & 0,01028 & 0,01024 \\
\hline $\mathbf{F}[\mathbf{k N}]$ & 1,375 & 1,372 & 1,329 & 1,330 \\
\hline
\end{tabular}

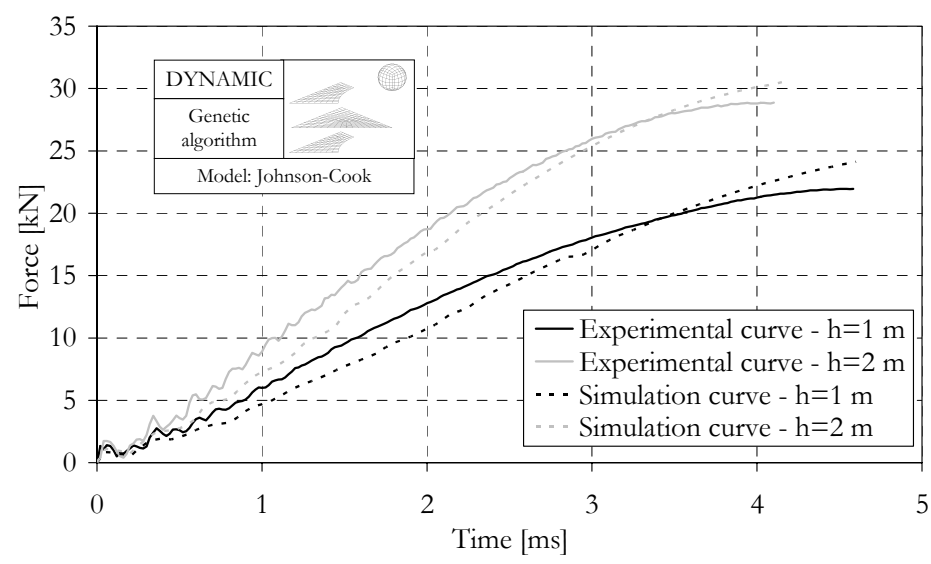

Figure 9: Comparison between experimental force-time curves of dynamic bending tests and simulations with Johnson-Cook model. 
Simple drop tower bending tests were performed at different loading speeds on specimen made of XE280P steel. Explicit simulations by means of FE models were run in order to identify the material strain rate characteristic parameters according to the Cowper-Symonds and the Johnson-Cook laws. The identification of static model parameters was also performed with the same algorithm and the same bending test at quasi-static loading conditions, but large identification uncertainty and drawbacks were encountered. A classical uniaxial tensile test was chosen for a more reliable and proper identification of static parameters and for the validation of the bending test modelling.

\section{Acknowledgement}

The authors kindly acknowledge the European Commission that, by funding the ADVANCE project through contract GRD1-200-25914, made this research activity possible.

\section{References}

[1] Jones, N., Structural Impact, Cambridge University Press, 1989.

[2] Symonds, P.S., Survey of methods of analysis for plastic deformation of structures under dynamic loading, Report No. BU/NSRDC, Brown University, 1967.

[3] Jones, N., Some comments on the modelling of material properties for dynamic structural plasticity, Proc. of Int. Conf. Mechanical Properties of Materials at High Rates of Strain, Oxford, pp. 435-445, 1989.

[4] Johnson, G.R. \& Cook, W.H., Fracture Characteristics of three metals subjected to various strains, strain rates, temperatures and pressures, Eng. Frac. Mech., Vol. 21, No. 1, pp. 31-48, 1985.

[5] Avalle, M., Belingardi, G., Vadori, R. \& Masciocco, G., Characterization of the strain rate sensitivity in the dynamic bending behavior of mild steel plates, Proc. EUROMAT 2000, Tours, pp. 505-510, 2000.

[6] Avalle, M., Belingardi, G. \& Gamarino, M., An inverse approach for the identification of strain-rate sensitivity parameters of sheet steels, Proc. SUSI 2004 (Structure under Shock and Impact), Creta (Grecia), pp.13-22, 27-29 March 2004. 